\section{Compartimentation de la réponse immunitaire chez le moustique Anopheles gambiae}

Conséquences pour une stratégie de lutte ciblant les vecteurs

Guillaume Carissimol,2, Emmanuel Bischoff ${ }^{1,2}$, Ken Vernick ${ }^{1,2,3}$
${ }^{1}$ Institut Pasteur, Unité de Génétique et Génomique des Insectes Vecteurs, 28, rue du Docteur Roux, 75015 Paris, France;

${ }^{2}$ CNRS, Unité Hôtes, Vecteurs et Pathogènes (URA3012), 28, rue du Docteur Roux, 75015 Paris, France ;

${ }^{3}$ Department of Microbiology, University of Minnesota, Minneapolis, MN, États-Unis.

guillaume.carissimo@gmail.com
> Les arbovirus possèdent un cycle biologique complexe; ils sont transmis à des hôtes mammifères (I'homme ou des animaux) par des arthropodes comme les moustiques, les mouches ou les tiques. L'incidence et les risques associés aux arbovirus augmentent dans le monde entier, et notamment en Europe occidentale et en Amérique du Nord où ces virus étaient jusqu'à présent absents. Parmi ces derniers, nous pouvons citer le virus de la dengue (famille des Flaviviridae) - avec $40 \%$ de la population mondiale qui vit dans les zones exposées -, ou le virus Chikungunya (famille des Togaviridae) qui a ré-émergé en 2006 sur l'île de La Réunion pour se répandre ensuite dans le monde entier. Tous deux sont transmis par des moustiques du genre Aedes.

La compréhension des interactions entre les pathogènes (bactéries, parasites et virus) et l'immunité que développe les insectes vecteurs (ou les nouveaux vecteurs potentiels favorisés par des changements environnementaux) vis-à-vis de ceux-ci, sont essentielles pour évaluer les risques associés à ces maladies et mettre en place des mesures de contrôle vectoriel adaptées. Une de ces mesures consiste à remplacer les moustiques du terrain par des moustiques résistants, en disséminant dans la nature des moustiques dont l'immunité envers un pathogène spécifique serait augmentée [1]. Dans ce contexte, nous avons évalué la réponse immunitaire contre le virus O'nyong nyong (famille des Togaviridae, virus le plus proche phylogénétiquement du Chikungunya [2]) du moustique Anopheles gambiae, principal vecteur du paludisme (maladie provoquée par un parasite eucaryote du genre Plasmodium) en Afrique, et nous l'avons comparée aux réponses antiparasitaires précédemment décrites dans la littérature [3] (certaines sont illustrées sur la Figure 1). Cette étude montre pour la première fois une compartimentation des réponses immunitaires dirigées contre les pathogènes chez ce moustique, ainsi que l'existence d'un équilibre entre les mécanismes complexes qui gouvernent la résistance ou la susceptibilité à divers pathogènes humains.

Les étapes de l'infection parasitaire et virale d'un moustique vecteur

Le paludisme est principalement transmis par Anopheles gambiae sur le continent africain. L'obtention du génome complet de ce moustique en 2002 [4] a permis de progresser dans la compréhension de sa réponse immunitaire contre le parasite Plasmodium [5].

Ce parasite infecte la femelle moustique lors d'un repas sanguin infectieux. La fécondation entre les gamètes matures du parasite se produit dans l'intestin du moustique et conduit à la formation d'une progéniture (ou zygote) qui se transforme rapidement en une forme mobile, l'ookinète. Ce stade parasitaire envahit l'épithélium digestif du moustique et s'insère entre les cellules épithéliales et la lame basale de l'intestin où l'ookinète se transforme en oocyste. L'oocyste va alors se développer pendant 14 jours environ avant de relâcher dans l'hémolymphe - l'équivalent du sang chez les arthropodes - qui baigne la cavité interne et tous les organes, des centaines de sporozoïtes (stade parasitaire infectant pour I'homme). Ces derniers peuvent ensuite envahir les glandes salivaires du moustique, et être transmis à un nouvel hôte lors du prochain repas sanguin du moustique. Trois barrières sont ainsi franchies: la barrière d'infection (épithélium intestinal) est traversée par l'ookinète, tandis que les sporozoïtes, en s'échappant de l'oocyste puis en envahissant les glandes salivaires, franchissent respectivement la barrière d'échappement intestinal puis la barrière de transmission (Figure 1). Lors de chacune de ces étapes, le parasite est confronté aux réponses immunitaires de l'hôte, et utilise différentes stratégies pour s'en protéger [5].

Dans le cas d'une infection virale d'un moustique, il est primordial de prendre en compte - comme on le fait pour une infection parasitaire -, le fait que le virus doit traverser plusieurs compartiments physiologiques avant de pouvoir être transmis à un nouvel hôte (Figure 1): il doit franchir la barrière d'infection intestinale, puis s'échapper de l'épithélium digestif pour entrer dans le compartiment systémique (la cavité interne baignée par l'hémolymphe) du moustique, enfin atteindre les glandes salivaires pour être transmis par la salive à un nouvel hôte. Il y a donc 


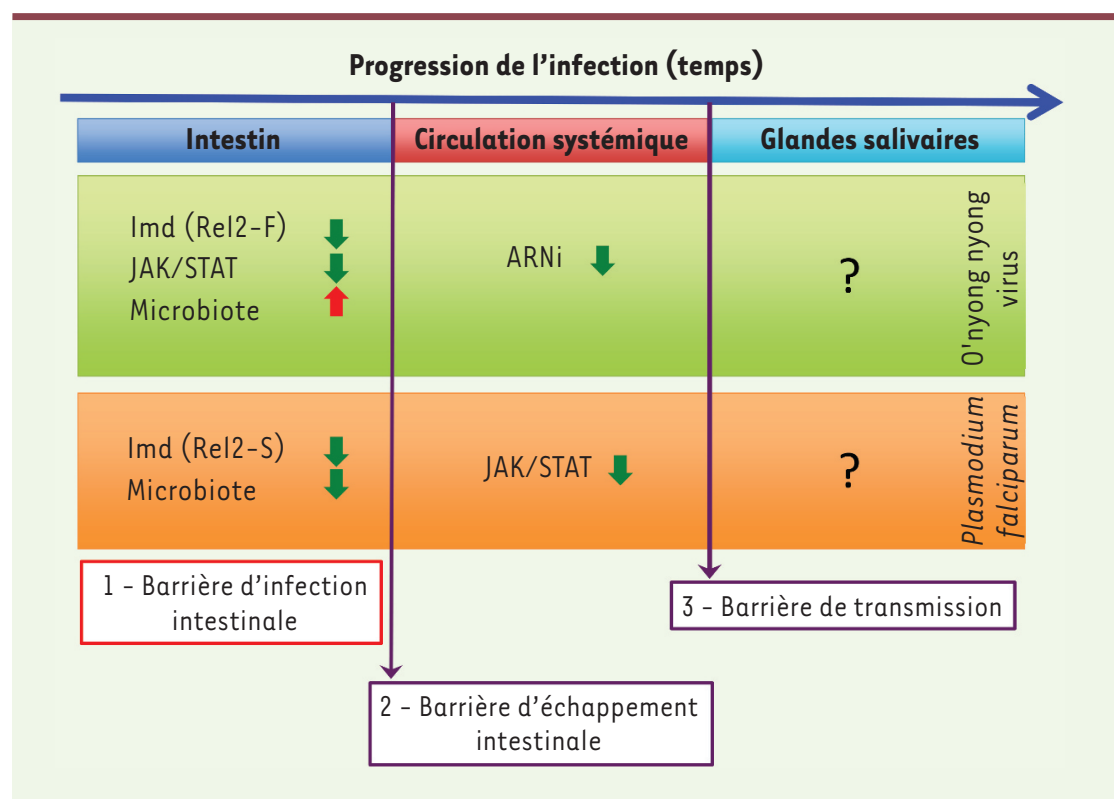

Figure 1. Cinétique d'infection et composants immunitaires impliqués dans les différents compartiments chez le moustique Anopheles gambiae. Infection par Plasmodium falciparum (cadre orange) et le virus 0 'nyong nyong (cadre vert) ; les flèches vertes indiquent la protection contre l'infection, les flèches rouges indiquent un effet facilitateur pour l'infection et les points d'interrogation indiquent les compartiments pour lesquels les composants immunitaires ne sont pas connus. Ce schéma illustre: (1) l'effet opposé du microbiote intestinal sur l'infection par deux pathogènes humains; (2) les différents acteurs transcriptionnels de la voie Imd impliqués dans l'immunité intestinale contre un virus et un parasite ; (3) le rôle antiviral ou antiparasitaire de la voie JAK/STAT en fonction des différents compartiments; et (4) la compartimentation de l'activité antivirale de l'ARN interférence.

une double composante, temporelle et spatiale, importante à considérer lors de l'étude des interactions du virus avec les réponses antivirales du moustique.

\section{Enjeux des compartiments immunitaires}

La réponse immunitaire intestinale, par sa précocité et sa localisation, est déterminante pour le devenir de l'infection du vecteur (cette étape étant responsable de la plus importante réduction du nombre de parasites lors de son cycle biologique) [6], tandis que la réponse systémique intervient dans le contrôle des niveaux d'infection pour la survie de l'insecte. Nous avons utilisé un virus fluorescent, génétiquement modifié pour produire la GFP (green fluorescent protein), pour établir la cinétique temporelle du cheminement du virus lors de l'infection du moustique Anopheles afin de déterminer quels sont les organes infectés et dans quel ordre ils le sont. Cela nous a permis de déterminer le moment adéquat pour évaluer la réponse antivirale intestinale, avant la dissémination du virus dans le reste de l'organisme. Lors des études précédentes sur l'immunité antivirale des moustiques Anopheles, le virus était directement injecté dans le thorax des insectes, comme cela se fait pour l'organisme modèle qu'est la drosophile [7-9], court-circuitant ainsi la barrière physique et immune que forme l'épithélium intestinal.

En comparant les réponses antivirales de l'intestin et du compartiment systémique (après injection du virus in vivo ou utilisation de lignées cellulaires), nous avons montré que, contrairement à ce qui était précédemment admis [7-9], I'ARN interférence (ARNi) ne joue pas de rôle antiviral dans l'intestin du moustique et n'intervient que secondairement dans le compartiment systémique, possiblement pour maintenir la réplication virale à des niveaux physiologiquement acceptables pour l'insecte. En fait, dans l'intestin du moustique, ce sont les voies Imd (immune deficiency) et JAK/STAT (Janus kinase/signal transducers and activators of transcription) qui contrôlent la réponse antivirale et déterminent donc si l'infection sera ou non productive, en empêchant - ou non - le virus de franchir la barrière intestinale pour avoir accès aux glandes salivaires. La compartimentation de la réponse immunitaire du moustique détermine donc les voies antivirales mises en jeu lors des différentes étapes d'infection.

\section{Rôle de la flore intestinale du} moustique dans l'immunité

Il est intéressant de noter que les voies Imd et JAK/STAT permettent aussi au moustique de contrôler l'homéostasie de sa flore bactérienne intestinale (ou microbiote). Récemment, plusieurs équipes de recherche différentes ont montré que le microbiote des Anopheles pouvait jouer un rôle dans la susceptibilité du moustique à l'infection par les parasites Plasmodium [10]. Quant à savoir si la flore intestinale agit directement sur les parasites ou sur l'immunité de l'épithélium digestif, cela est encore débattu. De nombreuses études montrent que la réduction de la flore intestinale par un traitement antibiotique augmente l'infection des Anopheles par Plasmodium, ce que nous avons vérifié avec les moustiques de notre laboratoire [3]. Mais, lorsque nous avons effectué la même expérience dans le cas d'une infection virale, nous avons observé l'effet inverse: la réduction de la flore intestinale diminue l'infection virale. Ces travaux démontrent donc que l'effet de la flore intestinale sur la susceptibilité des moustiques à une infection donnée dépend du pathogène considéré. Nous avons également montré, par des expériences de complémentation, que la présence de bactéries intestinales vivantes et en phase de croissance lors du repas infectieux était responsable de cet effet. 
Dans le futur

Notre étude souligne que des biais expérimentaux, comme l'injection directe de virus court-circuitant la voie d'ingestion habituelle, ne donnent qu'une image partielle des voies immunitaires mises en jeu lors de la réponse à un pathogène [3]. Dans le futur, il faudra également s'intéresser aux voies immunitaires contrôlant la barrière d'infection des glandes salivaires. En effet, modifier l'immunité pour produire des vecteurs résistant à une infection donnée pourrait avoir des effets différents, voire opposés, selon les méthodes utilisées et selon les compartiments de l'organisme ciblés. Préalablement à l'utilisation sur le terrain de moustiques modifiés, ce qui constitue une stratégie de contrôle vectoriel, l'effet des modifications sur l'immunité vis-à-vis de divers pathogènes devra être rigoureusement testé.
En effet, il serait dramatique que des moustiques censés être plus résistants à Plasmodium deviennent susceptibles à d'autres pathogènes. $\diamond$

Compartimentalization of immune responses in the mosquito Anopheles gambiae: consequences for insect vector immunity research

\section{LIENS D'INTÉRÊT}

Les auteurs déclarent n'avoir aucun lien d'intérêt concernant les données publiées dans cet article.

\section{REMERCIEMENTS}

Merci à Julie Reveillaud et Christelle Petit pour leur relecture critique.

\section{RÉFÉRENCES}

1. Wang S, Jacobs-Lorena M. Genetic approaches to interfere with malaria transmission by vector mosquitoes. Trends Biotechnol 2013 ; 31 : 185-93.

2. Powers AM, Brault AC, Shirako $Y$, et al. Evolutionary relationships and systematics of the alphaviruses. Virol $2001 ; 75$ : 10118-31.
3. Carissimo G, Pondeville E, McFarlane M, et al. Antiviral immunity of Anopheles gambiae is highly compartmentalized, with distinct roles for RNA interference and gut microbiota. Proc Natl Acad Sci USA $2015 ; 112$ : ع176-85.

4. Holt RA, Subramanian GM, Halpern A, et al. The genome sequence of the malaria mosquito Anopheles gambiae. Science 2002 ; 298 : 129-49.

5. Cirimotich CM, Dong Y, Garver LS, et al. Mosquito immune defenses against Plasmodium infection. Dev Comp Immunol 2010 ; 34 : 387-95.

6. Smith RC, Vega-Rodriguez J, Jacobs-Lorena M. The Plasmodium bottleneck: malaria parasite losses in the mosquito vector. Memorias do Instituto Oswaldo Cruz 2014; 109: 644-61.

7. Keene KM, Foy BD, Sanchez-Vargas I, et al. RNA interference acts as a natural antiviral response to O'nyong-nyong virus (Alphavirus; Togaviridae) infection of Anopheles gambiae. Proc Natl Acad Sci USA 2004 ; $101: 17240-5$

8. Waldock J, Olson KE, Christophides GK. Anopheles gambiae antiviral immune response to systemic O'nyong-nyong infection. PLoS Negl Trop Dis 2012 ; 6 : el565.

9. Myles KM, Wiley MR, Morazzani EM, Adelman ZN. Alphavirus-derived small RNAs modulate pathogenesis in disease vector mosquitoes. Proc Natl Acad Sci USA 2008 ; 105 : 19938-43.

10. Dennison NJ, Jupatanakul N, Dimopoulos G. The mosquito microbiota influences vector competence for human pathogens. Curr Opinion Insect Sci 2014 ; $3: 6-13$.

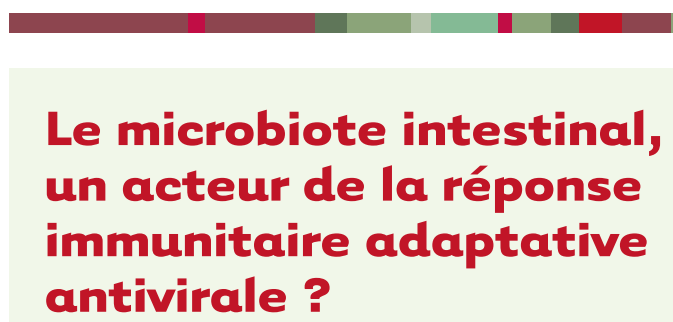

Benoit Chassaing

Une étude récente a permis de mettre en évidence une relation inattendue entre le microbiote intestinal et la réponse immunitaire adaptative faisant suite à une vaccination. En effet, cette étude démontre que la population de bactéries que nous hébergeons dans notre intestin joue un rôle majeur dans la capacité de notre système immunitaire à se protéger contre une infection virale.

\section{La grippe saisonnière}

Le virus de la grippe (influenza) est responsable d'infections respiratoires aiguës très contagieuses chez l'homme. Dans les pays à climat tempéré, il est responsable d'épidémies saisonnières qui surviennent au cours de l'hiver. Souvent considérée comme une maladie bénigne, la grippe est néanmoins à l'origine d'environ 250000 à 500000 décès par an, surtout chez les jeunes enfants de moins de deux ans et les personnes de plus de 65 ans. Il existe, comme pour de nombreux agents infectieux, des vaccins disponibles qui protègent efficacement contre la grippe. Cependant, le virus influenza est en perpétuelle évolution et de nombreuses mutations affectent sa structure. II faut donc se faire vacciner tous les ans, le vaccin proposé chaque année étant conçu pour com-
Institute for biomedical sciences, center for inflammation, immunity, and infection, Georgia State University, 100 Piedmont ave SE, Petit Science Center, room 708, Atlanta, GA 30303, États-Unis.

bchassaing@gsu.edu

chassaingbenoit@yahoo.fr

battre uniquement la souche de l'hiver à venir. L'efficacité du vaccin antigrippal dépend de plusieurs facteurs, dont la similitude entre la souche du vaccin et la souche infectieuse circulante, ainsi que l'âge et le statut immunologique de la personne vaccinée.

\section{Immunité innée et microbiote intestinal}

Afin de se protéger des agents infectieux, notre organisme met en place deux types de réponses immunitaires, innée et adaptative. La réponse immunitaire innée peut être immédiate et fait intervenir de nombreux acteurs cellulaires, par exemple 